\title{
Extended States for Polyharmonic Operators with Quasi-periodic Potentials in Dimension Two
}

\author{
Yu. Karpeshina, R. Shterenberg
}

\begin{abstract}
We consider a polyharmonic operator $H=(-\Delta)^{l}+V(\mathbf{x})$ in dimension two with $l \geq 2, l$ being an integer, and a quasi-periodic potential $V(\mathbf{x})$. We prove that the spectrum of $H$ contains a semiaxis and there is a family of generalized eigenfunctions at every point of this semiaxis with the following properties. First, the eigenfunctions are close to plane waves $e^{i\langle\mathbf{k}, \mathbf{x}\rangle}$ at the high energy region. Second, the isoenergetic curves in the space of momenta $\mathbf{k}$ corresponding to these eigenfunctions have a form of slightly distorted circles with holes (Cantor type structure). A new method of multiscale analysis in the momentum space is developed to prove these results.
\end{abstract}

We study an operator

$$
H=(-\Delta)^{l}+V(\mathbf{x})
$$

in dimension two, where $l$ is an integer, $l \geq 2, V(\mathbf{x})$ is a quasi-periodic potential being a trigonometric polynomial:

$$
V=\sum_{\mathbf{s}_{1}, \mathbf{s}_{2} \in \mathbb{Z}^{2}, 0<\left|\mathbf{s}_{1}\right|+\left|\mathbf{s}_{2}\right| \leq Q} V_{\mathbf{s}_{1}, \mathbf{s}_{2}} e^{2 \pi i\left\langle\mathbf{s}_{1}+\alpha \mathbf{s}_{2}, \mathbf{x}\right\rangle}, \quad 1 \leq Q<\infty .
$$

We assume that the irrationality measure $\mu$ of $\alpha$ is finite: $\mu<\infty$, or in other words, that $\alpha$ is not a Liouville number 1 .

The one-dimensional situation $d=1, l=1$ is thoroughly investigated in discrete and continuum settings, see e.g. [1]-[13] and references there. It is known that a one-dimensional quasi-periodic Schrödinger operator demonstrates spectral and transport properties which are not close to those of a periodic operator. The spectrum of the quasi-periodic operator is, as a rule, a Cantor set, while in the periodic case, it has a band structure. In the periodic case the spectrum is absolutely continuous, while in the quasiperiodic case, it can have any nature: absolutely continuous, singular continuous and pure point. The transition between different types of spectrum can happen even with a small

\footnotetext{
${ }^{1}$ Note, that $\mu \geq 2$ for any irrational number $\alpha$.
} 
change of a coefficient in a quasi-periodic operator [8]. The mechanism of the difference in spectral behavior between periodic and quasi-periodic cases can be explained by a phenomenon which is known as resonance tunneling in quantum mechanics. It is associated with small denominators appearing in formal series of perturbation theory. Since the spectrum of the one-dimensional Laplacian is thin (multiplicity 2), resonance tunneling can produce an effect strong enough to destroy the spectrum. If a potential is periodic, then resonance tunneling produces gaps in the spectrum near the points $\lambda_{n}=(\pi n / a)^{2}$, $n \in \mathbb{Z}, a$ being the period of the potential. If the potential is quasi-periodic, then it can be thought as a sort of combination of infinite number of periodic potentials, each of them producing gaps near its own $\lambda_{n}$-s. Since the set of all $\lambda_{n}$-s can be dense, the number of points surrounded by gaps can be dense too. Thus, the spectrum gets a Cantor like structure. The properties of the operator in the high energy region for the continuum case $d=1$ are studied in [1]-[4], [7]. The KAM method is used to prove absolute continuity of the spectrum and existence of quasiperiodic solutions at high energies.

There are important results on the density of states, spectrum, localization concerning the quasi-periodic operators in $\mathbb{Z}^{d}$ and, partially, in $\mathbb{R}^{d}, d>1$, e.g. [14]-[24]. However, it is still much less known about (1) then about its one-dimensional analog. The properties of the spectrum in the high energy region, existence of extended states and quantum transport are still wide open problems in the multidimensional case.

Here we study properties of the spectrum and eigenfunctions of (11) in the high energy region. We prove the following results for the case $d=2, l \geq 2$.

1. The spectrum of the operator (11) contains a semiaxis.

This is a generalization of a renown Bethe-Sommerfeld conjecture, which states that in the case of a periodic potential, $l=1$ and $d \geq 2$, the spectrum of (1) contains a semiaxis. There is a variety of proofs for the periodic case, the earliest one is 25]. For a limit-periodic periodic potential, being periodic in one direction, the conjecture is proved in [26]. For a general case of limit-periodic potential the conjecture is proven in [27]-[29]. Here we present the first proof of (a generalized) Bethe-Sommerfeld conjecture for a quasi-periodic potential.

2. There are generalized eigenfunctions $\Psi_{\infty}(\mathbf{k}, \mathbf{x})$, corresponding to the semi-axis, which are close to plane waves: for every $\mathbf{k}$ in an extensive subset $\mathcal{G}_{\infty}$ of $\mathbb{R}^{2}$, there is a solution $\Psi_{\infty}(\mathbf{k}, \mathbf{x})$ of the equation $H \Psi_{\infty}=\lambda_{\infty} \Psi_{\infty}$ which can be described by the formula:

$$
\begin{gathered}
\Psi_{\infty}(\mathbf{k}, \mathbf{x})=e^{i\langle\mathbf{k}, \mathbf{x}\rangle}\left(1+u_{\infty}(\mathbf{k}, \mathbf{x})\right) \\
\left\|u_{\infty}\right\|_{L_{\infty}\left(\mathbb{R}^{2}\right)}=\underset{|\mathbf{k}| \rightarrow \infty}{=} O\left(|\mathbf{k}|^{-\gamma_{1}}\right), \quad \gamma_{1}>0
\end{gathered}
$$

where $u_{\infty}(\mathbf{k}, \mathbf{x})$ is a quasi-periodic function, namely a point-wise convergent series of exponentials $e^{i\langle\mathbf{n}+\alpha \mathbf{m}, \mathbf{x}\rangle}, \mathbf{n}, \mathbf{m} \in \mathbb{Z}^{2}$. The eigenvalue $\lambda_{\infty}(\mathbf{k})$, corresponding to 
$\Psi_{\infty}(\mathbf{k}, \mathbf{x})$, is close to $|\mathbf{k}|^{2 l}$ :

$$
\lambda_{\infty}(\mathbf{k}) \underset{|\mathbf{k}| \rightarrow \infty}{=}|\mathbf{k}|^{2 l}+O\left(|\mathbf{k}|^{-\gamma_{2}}\right), \quad \gamma_{2}>0 .
$$

The "non-resonant" set $\mathcal{G}_{\infty}$ of vectors $\mathbf{k}$, for which (3) - (5) hold, is an extensive Cantor type set: $\mathcal{G}_{\infty}=\cap_{n=1}^{\infty} \mathcal{G}_{n}$, where $\left\{\mathcal{G}_{n}\right\}_{n=1}^{\infty}$ is a decreasing sequence of sets in $\mathbb{R}^{2}$. Each $\mathcal{G}_{n}$ has a finite number of holes in each bounded region. More and more holes appear when $n$ increases, however holes added at each step are of smaller and smaller size. The set $\mathcal{G}_{\infty}$ satisfies the estimate:

$$
\left|\mathcal{G}_{\infty} \cap \mathbf{B}_{\mathbf{R}}\right| \underset{R \rightarrow \infty}{=}\left|\mathbf{B}_{\mathbf{R}}\right|\left(1+O\left(R^{-\gamma_{3}}\right)\right), \quad \gamma_{3}>0
$$

where $\mathbf{B}_{\mathbf{R}}$ is the disk of radius $R$ centered at the origin, $|\cdot|$ is the Lebesgue measure in $\mathbb{R}^{2}$.

3. The set $\mathcal{D}_{\infty}(\lambda)$, defined as a level (isoenergetic) set for $\lambda_{\infty}(\mathbf{k})$,

$$
\mathcal{D}_{\infty}(\lambda)=\left\{\mathbf{k} \in \mathcal{G}_{\infty}: \lambda_{\infty}(\mathbf{k})=\lambda\right\}
$$

is proven to be a slightly distorted circle with infinite number of holes. It can be described by the formula:

$$
\mathcal{D}_{\infty}(\lambda)=\left\{\mathbf{k}: \mathbf{k}=\varkappa_{\infty}(\lambda, \vec{\nu}) \vec{\nu}, \vec{\nu} \in \mathcal{B}_{\infty}(\lambda)\right\}
$$

where $\mathcal{B}_{\infty}(\lambda)$ is a subset of the unit circle $S_{1}$. The set $\mathcal{B}_{\infty}(\lambda)$ can be interpreted as the set of possible directions of propagation for almost plane waves (3). The set $\mathcal{B}_{\infty}(\lambda)$ has a Cantor type structure and an asymptotically full measure on $S_{1}$ as $\lambda \rightarrow \infty$ :

$$
L\left(\mathcal{B}_{\infty}(\lambda)\right) \underset{\lambda \rightarrow \infty}{=} 2 \pi+O\left(\lambda^{-\gamma_{4} / 2 l}\right), \quad \gamma_{4}>0,
$$

here and below $L(\cdot)$ is a length of a curve. The value $\varkappa_{\infty}(\lambda, \vec{\nu})$ in (7) is the "radius" of $\mathcal{D}_{\infty}(\lambda)$ in a direction $\vec{\nu}$. The function $\varkappa_{\infty}(\lambda, \vec{\nu})-\lambda^{1 / 2 l}$ describes the deviation of $\mathcal{D}_{\infty}(\lambda)$ from the perfect circle of the radius $\lambda^{1 / 2 l}$. It is proven that the deviation is asymptotically small:

$$
\varkappa_{\infty}(\lambda, \vec{\nu}) \underset{\lambda \rightarrow \infty}{=} \lambda^{1 / 2 l}+O\left(\lambda^{-\gamma_{5}}\right), \quad \gamma_{5}>0
$$

4. The branch of the spectrum corresponding to $\Psi_{\infty}(\mathbf{k}, \mathbf{x})$ (the semiaxis) is absolutely continuous.

To prove the results listed above we suggest a method which can be described as multiscale analysis in the space of momenta. This is a development of the method, which is used in [27]-[29] for the case of limit-periodic potentials. The essential difference is that 
in [27]-29] we constructed a modification of KAM method, where the space variable $\mathbf{x}$ still plays some role (e.g. in the uniform in $\mathbf{x}$ approximation of a limit-periodic potential by periodic ones), while in the present situation all considerations are happening in the space of the dual variable $\mathbf{k}$. The KAM method in [27]-[29] is motivated by [30]-[32], where the method is used for periodic problems. Multiscale analisys which we apply here is deeply analogous to the original multiscale method developed in [33] (see also [19], [20]) for the proof of localization. The essential difference is that in [33], [19], [20] the multiscale procedure is constructed with respect to space variable $\mathbf{x}$ to prove localization, while we construct a multiscale procedure in the space of momenta $\mathbf{k}$ to prove delocalization.

Here is a brief description of the iteration procedure which leads to the results described above. Indeed, let $\mathbf{k} \in \mathbb{R}^{2}$. We consider a set of finite linear combinations of plane waves $e^{i\langle\mathbf{k}+\mathbf{p}+\alpha \mathbf{m}, \mathbf{x}\rangle}, \mathbf{p}, \mathbf{m} \in \mathbb{Z}^{2}$. The set is invariant under action of the differential expression (11). Let $H(\mathbf{k})$ be a matrix describing action of (11) in the linear set of the exponentials. Obviously,

$$
\begin{gathered}
H(\mathbf{k})=H_{0}(\mathbf{k})+V, \quad H_{0}(\mathbf{k})_{(\mathbf{p}, \mathbf{m}),\left(\mathbf{p}^{\prime}, \mathbf{m}^{\prime}\right)}=|\mathbf{k}+\mathbf{p}+\alpha \mathbf{m}|_{\mathbb{R}^{2}}^{2 l} \delta_{\left(\mathbf{p}, \mathbf{p}^{\prime}\right)} \delta_{\left(\mathbf{m}, \mathbf{m}^{\prime}\right)}, \\
V_{(\mathbf{p}, \mathbf{m}),\left(\mathbf{p}^{\prime}, \mathbf{m}^{\prime}\right)}=V_{\mathbf{p}-\mathbf{p}^{\prime}, \mathbf{m}-\mathbf{m}^{\prime}}
\end{gathered}
$$

Next, we consider an expanding sequence of finite sets $M_{n}$ in the space $\mathbb{Z}^{2} \times \mathbb{Z}^{2}$ of indices $(\mathbf{p}, \mathbf{m}): M_{n} \subset M_{n+1}, \lim _{n \rightarrow \infty} M_{n}=\mathbb{Z}^{2} \times \mathbb{Z}^{2}$. Let $P_{n}$ be the characteristic projection of set $M_{n}$ in the space $\ell^{2}\left(\mathbb{Z}^{2} \times \mathbb{Z}^{2}\right)$. We consider a sequence of finite matrices $H^{(n)}(\mathbf{k})=$ $P_{n} H(\mathbf{k}) P_{n}$. Each matrix corresponds to a finite dimensional operator in $\ell^{2}\left(\mathbb{Z}^{2} \times \mathbb{Z}^{2}\right)$, given that the operator acts as zero on $\left(I-P_{n}\right) \ell^{2}$. For each $n$ we construct a "non-resonant" set $\mathcal{G}_{n}$ in the space $\mathbb{R}^{2}$ of momenta $\mathbf{k}$, such that: if $\mathbf{k} \in \mathcal{G}_{n}$, then $H^{(n)}(\mathbf{k})=P_{n} H(\mathbf{k}) P_{n}$ has an eigenvalue $\lambda_{n}(\mathbf{k})$ and its spectral projector $\mathcal{E}_{n}(\mathbf{k})$ which can be described by perturbation formulas with respect to the previous operator $H^{(n-1)}(\mathbf{k})$. If $\mathbf{k} \in \cap_{n=1}^{\infty} \mathcal{G}_{n}$ then $\lambda_{n}(\mathbf{k})$ and $\varepsilon_{n}(\mathbf{k})$ have limits. The linear combinations of the exponentials, corresponding to the projectors $\mathcal{E}_{n}(\mathbf{k})$, have a point-wise limit in $\mathbf{x}$, the limit being a generalized eigenfunction of (11). The generalized eigenfunction is close to the plane wave $e^{i<\mathbf{k}, \mathbf{x}>}$ in the high energy region.

Each matrix $H^{(n)}$ is considered as a perturbation of a matrix $\hat{H}^{(n)}$, the latter has a block structure, i.e., consists of a variety of blocks $H^{(s)}(\mathbf{k}+\mathbf{p}+\alpha \mathbf{m}), s=1, \ldots, n-1$, and, naturally, some diagonal terms. Blocks with different indices $(s)$ have sizes of different orders of magnitude (the size increasing with $s$ ). Thus we have a multiscale structure in the definition of $\hat{H}^{(n)}$. We use $\hat{H}^{(n)}(\mathbf{k})$ as a starting operator to construct perturbation series for $H^{(n)}(\mathbf{k})$. At a step $n$ we apply our knowledge of spectral properties of $H^{(s)}(\mathbf{k}+$ $\left.\mathbf{p}^{\prime}+\alpha \mathbf{m}^{\prime}\right), s=1, \ldots, n-1, \mathbf{p}^{\prime}, \mathbf{m}^{\prime} \in \mathbb{Z}^{2}$, obtained in the previous steps, to describe spectral properties of $H^{(n)}(\mathbf{k}+\mathbf{p}+\alpha \mathbf{m}), \mathbf{p}, \mathbf{m} \in \mathbb{Z}^{2}$ and to construct $\mathcal{G}_{n}$.

At step one we use a regular perturbation theory and elementary geometric considerations to prove the following results. There is a set $\mathcal{G}_{1} \subset \mathbb{R}^{2}$ such that: if $\mathbf{k} \in \mathcal{G}_{1}$, then 
the operator $H^{(1)}(\mathbf{k})$ has a single eigenvalue close to the unperturbed one:

$$
\lambda^{(1)}(\mathbf{k}) \underset{|\mathbf{k}| \rightarrow \infty}{=}|\mathbf{k}|^{2 l}+O\left(|\mathbf{k}|^{-\gamma_{2}}\right), \quad \gamma_{2}>0 .
$$

A normalized eigenvector $\mathbf{u}^{(1)}$ is also close to the unperturbed one: $\mathbf{u}^{(1)}=\mathbf{u}^{(0)}+\tilde{\mathbf{u}}^{(1)}$, where $\left(\mathbf{u}^{(0)}\right)_{(\mathbf{p}, \mathbf{m})}=\delta_{\mathbf{p}, \mathbf{0}} \delta_{\mathbf{m}, \mathbf{0}}$ and the $l^{1}$-norm of $\tilde{\mathbf{u}}^{(1)}$ is small: $\left\|\tilde{\mathbf{u}}^{(1)}\right\|_{l^{1}}<|\mathbf{k}|^{-\gamma_{1}}, \gamma_{1}>0$. It follows that:

$$
\Psi_{1}(\mathbf{k}, \mathbf{x})=e^{i\langle\mathbf{k}, \mathbf{x}\rangle}+\tilde{u}_{1}(\mathbf{k}, \mathbf{x}), \quad\left\|\tilde{u}_{1}\right\|_{L_{\infty}\left(\mathbb{R}^{2}\right)} \underset{|\mathbf{k}| \rightarrow \infty}{=} O\left(|\mathbf{k}|^{-\gamma_{1}}\right), \quad \gamma_{1}>0,
$$

where $\Psi_{1}(\mathbf{k}, \mathbf{x}), \tilde{u}_{1}(\mathbf{k}, \mathbf{x})$ are the linear combinations of the exponentials corresponding to vectors $\mathbf{u}^{(1)}$ and $\tilde{\mathbf{u}}^{(1)}$, respectively. It is shown that function $\Psi_{1}(\mathbf{k}, \mathbf{x})$ satisfies the equation for eigenfunctions with a good accuracy:

$$
-\Delta \Psi_{1}+V \Psi_{1}=|\mathbf{k}|^{2 l} \Psi_{1}+f_{1}, \quad\left\|f_{1}\right\|_{L_{\infty}\left(\mathbb{R}^{2}\right)} \underset{|\mathbf{k}| \rightarrow \infty}{=} O\left(|\mathbf{k}|^{-\gamma_{6}}\right) \quad \gamma_{6}>0 .
$$

Relation (10) is differentiable:

$$
\nabla \lambda^{(1)}(\mathbf{k}) \underset{|\mathbf{k}| \rightarrow \infty}{=} 2 l|\mathbf{k}|^{2 l-2} \mathbf{k}+O\left(|\mathbf{k}|^{-\gamma_{7}}\right), \quad \gamma_{7}>0 .
$$

Next, we construct a sequence $\mathcal{G}_{n}, n \geq 2$, such for any $\mathbf{k} \in \mathcal{G}_{n}$ the operator $H^{(n)}(\mathbf{k})$ has a single eigenvalue $\lambda^{(n)}(\mathbf{k})$ in a super exponentially small neighborhood of $\lambda^{(n-1)}(\mathbf{k})$ :

$$
\lambda^{(n)}(\mathbf{k}) \underset{|\mathbf{k}| \rightarrow \infty}{=} \lambda^{(n-1)}(\mathbf{k})+O\left(|\mathbf{k}|^{-|\mathbf{k}|^{\gamma_{8} n}}\right), \quad \gamma_{8}>0 .
$$

Similar estimates hold for the eigenvectors and the corresponding functions $\Psi_{n}(\mathbf{k}, \mathbf{x})$ :

$$
\begin{array}{lll}
\Psi_{n}(\mathbf{k}, \mathbf{x})=\Psi_{n-1}(\mathbf{k}, \mathbf{x})+\tilde{u}_{n}(\mathbf{k}, \mathbf{x}), \quad\left\|\tilde{u}_{n}\right\|_{L_{\infty}\left(\mathbb{R}^{2}\right)} \underset{|\mathbf{k}| \rightarrow \infty}{=} O\left(|\mathbf{k}|^{-|\mathbf{k}|^{\gamma_{9} n}}\right), & \gamma_{9}>0 . \\
-\Delta \Psi_{n}+V \Psi_{n}=\lambda^{(n)}(\mathbf{k}) \Psi_{n}+f_{n}, \quad\left\|f_{n}\right\|_{L_{\infty}\left(\mathbb{R}^{2}\right)} \underset{|\mathbf{k}| \rightarrow \infty}{=} O\left(|\mathbf{k}|^{-|\mathbf{k}|^{\gamma_{1} 0^{n}}}\right), & \gamma_{10}>0 .
\end{array}
$$

Formula (14) is differentiable with respect to $\mathbf{k}$ :

$$
\nabla \lambda^{(n)}(\mathbf{k}) \underset{|\mathbf{k}| \rightarrow \infty}{=} \nabla \lambda^{(n-1)}(\mathbf{k})+O\left(|\mathbf{k}|^{-|\mathbf{k}|^{\gamma_{8} n}}\right), \quad \gamma_{8}>0 .
$$

In fact, for large $n$ estimates (14) - (17) are even stronger. The non-resonant set $\mathcal{G}_{n}$ is proven to be extensive in $\mathbb{R}^{2}$ :

$$
\left|\mathcal{G}_{n} \cap \mathbf{B}_{\mathbf{R}}\right| \underset{R \rightarrow \infty}{=}\left|\mathbf{B}_{\mathbf{R}}\right|\left(1+O\left(R^{-\gamma_{3}}\right)\right) .
$$

Estimates (14) - (18) are uniform in $n$. 


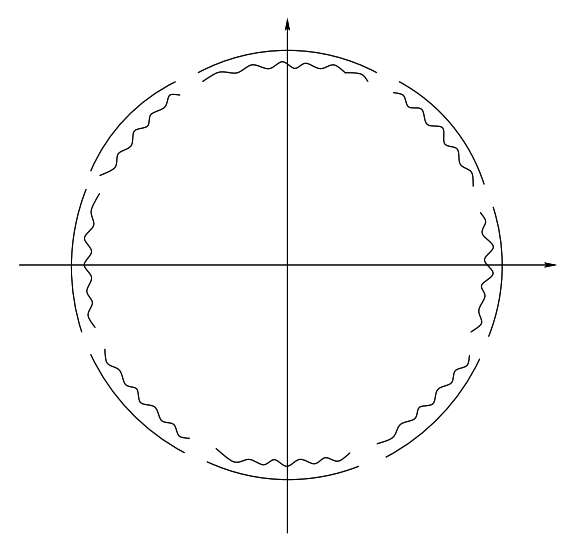

Figure 1: Isoenergetic curve $\mathcal{D}_{1}(\lambda)$

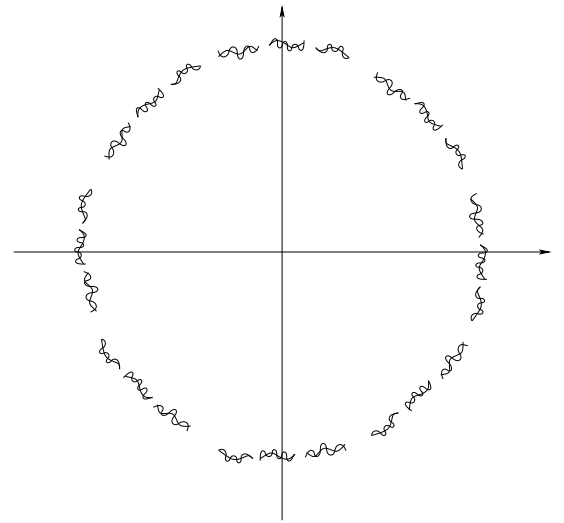

Figure 2: Isoenergetic curve $\mathcal{D}_{2}(\lambda)$

The set $\mathcal{D}_{n}(\lambda)$ is defined as the level (isoenergetic) set for the non-resonant eigenvalue $\lambda^{(n)}(\mathbf{k})$ :

$$
\mathcal{D}_{n}(\lambda)=\left\{\mathbf{k} \in \mathcal{G}_{n}: \lambda^{(n)}(\mathbf{k})=\lambda\right\} .
$$

This set is proven to be a slightly distorted circle with a finite number of holes (see Fig. 1. 21). The set $\mathcal{D}_{n}(\lambda)$ can be described by the formula:

$$
\mathcal{D}_{n}(\lambda)=\left\{\mathbf{k}: \mathbf{k}=\varkappa_{n}(\lambda, \vec{\nu}) \vec{\nu}, \vec{\nu} \in \mathcal{B}_{n}(\lambda)\right\},
$$

where $\mathcal{B}_{n}(\lambda)$ is a subset of the unit circle $S_{1}$. The set $\mathcal{B}_{n}(\lambda)$ can be interpreted as the set of possible directions of propagation for almost plane waves $\Psi_{n}(\mathbf{k}, \mathbf{x})$, see (11), (15). It has an asymptotically full measure on $S_{1}$ as $\lambda \rightarrow \infty$ :

$$
L\left(\mathcal{B}_{n}(\lambda)\right) \underset{\lambda \rightarrow \infty}{=} 2 \pi+O\left(\lambda^{-\gamma_{4} / 2 l}\right) .
$$

Each set $\mathcal{B}_{n}(\lambda)$ has only a finite number of holes, however their number is growing with $n$. More and more holes of a smaller and smaller size are added at each step. The value $\varkappa_{n}(\lambda, \vec{\nu})-\lambda^{1 / 2 l}$ gives the deviation of $\mathcal{D}_{n}(\lambda)$ from the perfect circle of the radius $\lambda^{1 / 2 l}$ in the direction $\vec{\nu}$. It is proven that the deviation is asymptotically small:

$$
\varkappa_{n}(\lambda, \vec{\nu})=\lambda^{1 / 2 l}+O\left(\lambda^{-\gamma_{5}}\right), \quad \frac{\partial \varkappa_{n}(\lambda, \vec{\nu})}{\partial \varphi}=O\left(\lambda^{-\gamma_{11}}\right), \quad \gamma_{5}, \gamma_{11}>0,
$$

$\varphi$ being an angle variable, $\vec{\nu}=(\cos \varphi, \sin \varphi)$. Estimates (20), (21) are uniform in $n$.

On each step more and more points are excluded from the non-resonant sets $\mathcal{G}_{n}$, thus $\left\{\mathcal{G}_{n}\right\}_{n=1}^{\infty}$ is a decreasing sequence of sets. The set $\mathcal{G}_{\infty}$ is defined as the limit set: $\mathcal{G}_{\infty}=\cap_{n=1}^{\infty} \mathcal{G}_{n}$. It has an infinite number of holes, but nevertheless satisfies the relation (6). For every $\mathbf{k} \in \mathcal{G}_{\infty}$ and every $n$, there is a generalized eigenfunction of $H^{(n)}$ of the type (11), (15)). It is proven that the sequence of $\Psi_{n}(\mathbf{k}, \mathbf{x})$ has a limit in $L_{\infty}\left(\mathbb{R}^{2}\right)$ when 
$\mathbf{k} \in \mathcal{G}_{\infty}$. The function $\Psi_{\infty}(\mathbf{k}, \mathbf{x})=\lim _{n \rightarrow \infty} \Psi_{n}(\mathbf{k}, \mathbf{x})$ is a generalized eigenfunction of $H$. It can be written in the form (3) - (44). Naturally, the corresponding eigenvalue $\lambda_{\infty}(\mathbf{k})$ is the limit of $\lambda^{(n)}(\mathbf{k})$ as $n \rightarrow \infty$.

It is shown that $\left\{\mathcal{B}_{n}(\lambda)\right\}_{n=1}^{\infty}$ is a decreasing sequence of sets, on each step more and more directions being excluded. We consider the limit $\mathcal{B}_{\infty}(\lambda)$ of $\mathcal{B}_{n}(\lambda)$ :

$$
\mathcal{B}_{\infty}(\lambda)=\bigcap_{n=1}^{\infty} \mathcal{B}_{n}(\lambda)
$$

This set has a Cantor type structure on the unit circle. It is proven that $\mathcal{B}_{\infty}(\lambda)$ has an asymptotically full measure on the unit circle (see (8) ). We prove that the sequence $\varkappa_{n}(\lambda, \vec{\nu}), n=1,2, \ldots$, describing the isoenergetic curves $\mathcal{D}_{n}(\lambda)$, quickly converges as $n \rightarrow$ $\infty$. We show that $\mathcal{D}_{\infty}(\lambda)$ can be described as the limit of $\mathcal{D}_{n}(\lambda)$ in the sense (7), where $\varkappa_{\infty}(\lambda, \vec{\nu})=\lim _{n \rightarrow \infty} \varkappa_{n}(\lambda, \vec{\nu})$ for every $\vec{\nu} \in \mathcal{B}_{\infty}(\lambda)$. It is shown that the derivatives of the functions $\varkappa_{n}(\lambda, \vec{\nu})$ (with respect to the angle variable on the unit circle) have a limit as $n \rightarrow \infty$ for every $\vec{\nu} \in \mathcal{B}_{\infty}(\lambda)$. We denote this limit by $\frac{\partial \varkappa_{\infty}(\lambda, \vec{\nu})}{\partial \varphi}$. Using (21), we prove that

$$
\frac{\partial \varkappa_{\infty}(\lambda, \vec{\nu})}{\partial \varphi}=O\left(\lambda^{-\gamma_{11}}\right) .
$$

Thus, the limit curve $\mathcal{D}_{\infty}(\lambda)$ has a tangent vector in spite of its Cantor type structure, the tangent vector being the limit of corresponding tangent vectors for $\mathcal{D}_{n}(\lambda)$ as $n \rightarrow \infty$. The curve $\mathcal{D}_{\infty}(\lambda)$ looks as a slightly distorted circle with infinite number of holes for every sufficiently large $\lambda, \lambda>\lambda_{*}(V)$. It immediately follows that $\left[\lambda_{*}, \infty\right)$ is in the spectrum of $H$ (Bethe-Sommerfeld conjecture).

The main technical difficulty to overcome is the construction of non-resonant sets $\mathcal{B}_{n}(\lambda)$ for every fixed sufficiently large $\lambda, \lambda>\lambda_{0}(V)$, where $\lambda_{0}(V)$ is the same for all $n$. The set $\mathcal{B}_{n}(\lambda)$ is obtained by deleting a "resonant" part from $\mathcal{B}_{n-1}(\lambda)$. Definition of $\mathcal{B}_{n-1}(\lambda) \backslash$ $\mathcal{B}_{n}(\lambda)$ includes eigenvalues of $H^{(n-1)}(\mathbf{k})$. To describe $\mathcal{B}_{n-1}(\lambda) \backslash \mathcal{B}_{n}(\lambda)$ one has to consider not only non-resonant eigenvalues of the type (10), (14), but also resonant eigenvalues, for which no suitable formulas are known. Absence of formulas causes difficulties in estimating the size of $\mathcal{B}_{n-1}(\lambda) \backslash \mathcal{B}_{n}(\lambda)$. To treat this problem we start with introducing an angle variable $\varphi \in[0,2 \pi), \vec{\nu}=(\cos \varphi, \sin \varphi) \in S_{1}$ and consider sets $\mathcal{B}_{n}(\lambda)$ in terms of this variable. Next, we show that the resonant set $\mathcal{B}_{n-1}(\lambda) \backslash \mathcal{B}_{n}(\lambda)$ can be described as the set of zeros of functions of the type

$$
\operatorname{det}\left(H^{(s)}\left(\vec{\varkappa}_{n-1}(\varphi)+\mathbf{p}+\alpha \mathbf{m}\right)-\lambda-\varepsilon\right), \quad s=1, \ldots, n-1, \quad(\mathbf{p}, \mathbf{m}) \in M_{n} \backslash(\mathbf{0}, \mathbf{0}),
$$

where $\vec{\varkappa}_{n-1}(\varphi)$ is a vector-function describing $\mathcal{D}_{n-1}(\lambda): \vec{\varkappa}_{n-1}(\varphi)=\varkappa_{n-1}(\lambda, \vec{\nu}) \vec{\nu}$. To obtain $\mathcal{B}_{n-1}(\lambda) \backslash \mathcal{B}_{n}(\lambda)$ we take all values of $\varepsilon$ in a small interval and $(\mathbf{p}, \mathbf{m})$ in some subset of $M_{n}$. Further, we extend our considerations to a complex neighborhood $\Phi_{0}$ of $[0,2 \pi)$. We show that the determinants are analytic functions of $\varphi$ and, by this, reduce the problem of 


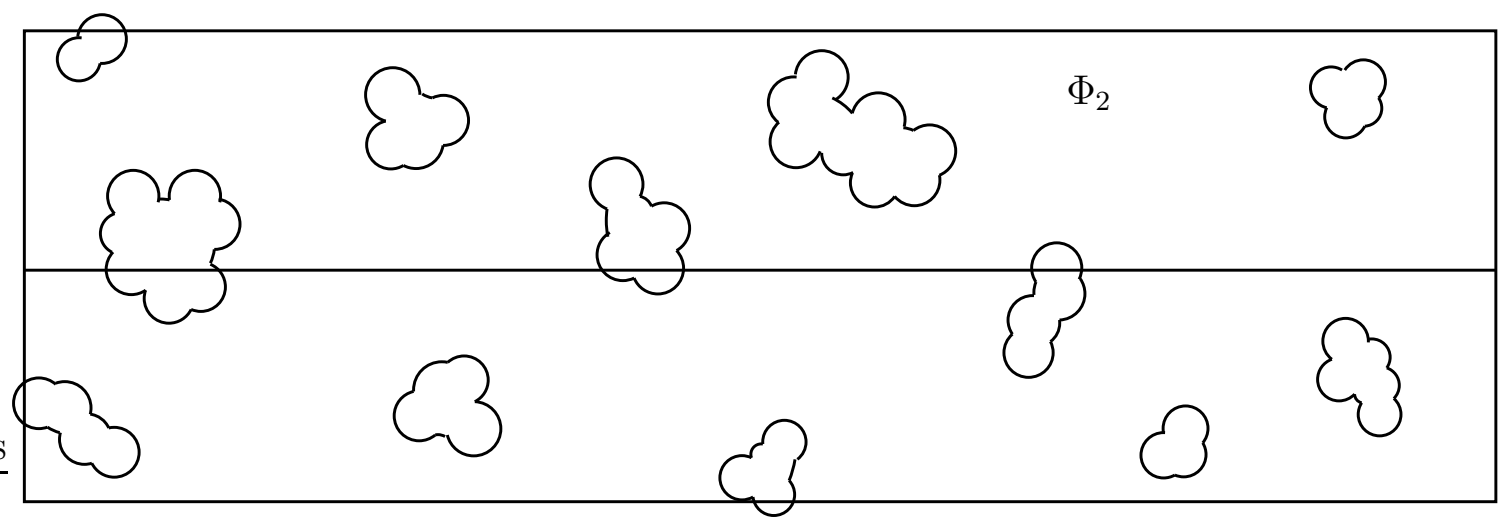

Figure 3: Set $\Phi_{2}$

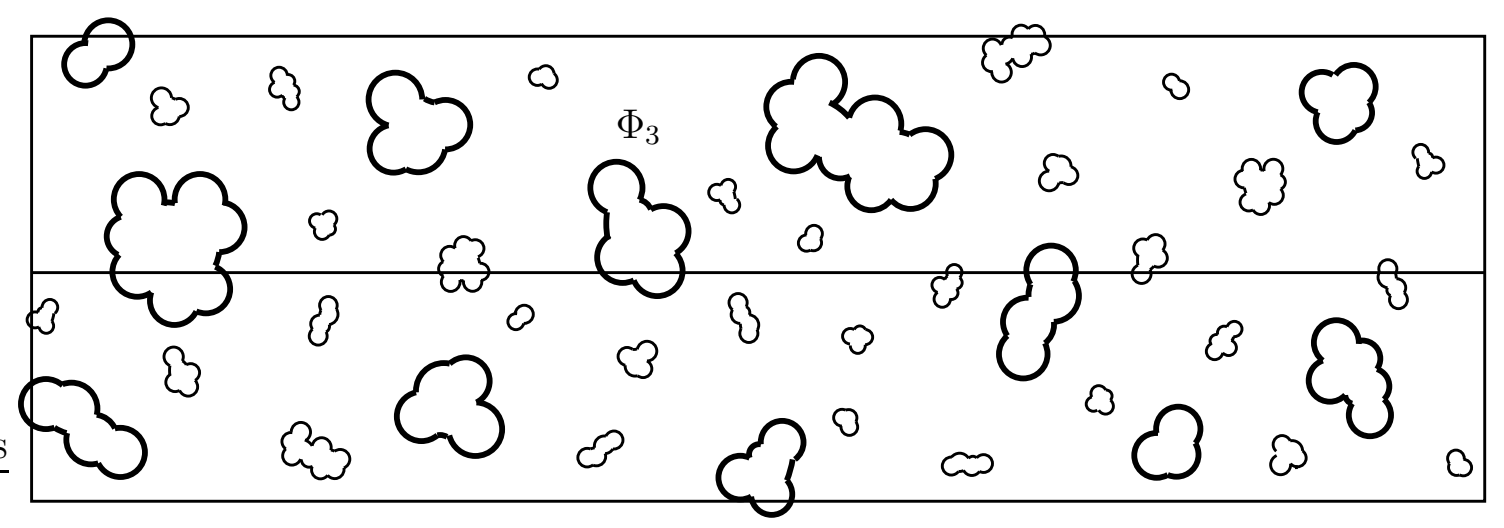

Figure 4: Set $\Phi_{3}$

estimating the size of the resonant set to a problem in complex analysis. We use theorems for analytic functions to count zeros of the determinants and to investigate how far the zeros move when $\varepsilon$ changes. It enables us to estimate the size of the zero set of the determinants, and, hence, the size of the non-resonant set $\Phi_{n} \subset \Phi_{0}$, which is defined as a non-zero set for the determinants. Proving that the non-resonant set $\Phi_{n}$ is sufficiently large, we obtain estimates (18) for $\mathcal{G}_{n}$ and (20) for $\mathcal{B}_{n}$, the set $\mathcal{B}_{n}$ corresponding to the real part of $\Phi_{n}$. To obtain $\Phi_{n}$ we delete from $\Phi_{0}$ more and more discs (holes) of smaller and smaller radii at each step. Thus, the non-resonant set $\Phi_{n} \subset \Phi_{0}$ has a structure of Swiss Cheese (Fig. 3, 44). Deleting a resonance set from $\Phi_{0}$ at each step of the recurrent procedure we call a "Swiss Cheese Method". The essential difference of our method from constructions of non-resonant sets in similar situations before (see e.g. [30]-[32], [34]) is that we construct a non-resonant set not only in the whole space of a parameter $\left(\mathbf{k} \in \mathbb{R}^{2}\right.$ here), but also on isoenergetic curves $\mathcal{D}_{n}(\lambda)$ in the space of the parameter, when $\lambda$ is sufficiently large. Estimates for the size of non-resonant sets on a curve require more 
subtle technical considerations than those sufficient for description of a non-resonant set in the whole space of the parameter. But as a reward, such estimates enable us to show that every isoenergetic set for $\lambda>\lambda_{0}$ is not empty and thus, to prove Bethe-Sommerfeld conjecture.

Note that generalization of the results from the case $l>1, l$ being an integer, to the case of rational $l$ satisfying the same inequality is relatively simple; it requires just slightly more careful technical considerations. The restriction $l>1$ is also technical, though it is more difficult to lift. The condition $l>1$ is needed only for the second step of the recurrent procedure. The authors plan to consider the case $l=1$ in a forthcoming paper. The requirement $\mu<\infty$ is essential, since we use it to estimate the minimal values of $|\mathbf{p}+\alpha \mathbf{m}|$ when $(\mathbf{p}, \mathbf{m}) \in M_{n} \backslash(\mathbf{0}, \mathbf{0})$. Such estimates are necessary for controlling small denominators in the perturbation series at each step.

Acknowledgement The authors are very grateful to Prof. Leonid Parnovski for useful discussions and to Prof. Young-Ran Lee for allowing us to use figures 1-4 from [27].

\section{References}

[1] E.I. Dinaburg, Ya. Sinai, The One-dimensional Schrödinger Equation with a Quasiperiodic Potential, Funct. Anal. Appl. 9 (1975), 279-289.

[2] H. Rûssmann, On the one dimensional Schrödinger equation with a quasi-periodic potential, Ann. N. Y. Acad. Sci. 357 (1980), 90-107.

[3] R. Johnson, J. Moser, The rotation number for almost periodic potentials, Commun. Math. Phys. 84 (1982), 403-438.

[4] J. Moser, J. Püschel, An extension of a result by Dinaburg and Sinai on quasiperiodic potentials, Comment. Math. Helvetic 59 (1984), 39-85.

[5] H.L. Cycon, R.G. Froese, W. Kirsch, B. Simon, Schrödinger Operators, Berlin: Springer Verlag, 1987, corrected and extended 2nd printing, Springer Verlag, 2008.

[6] L. Pastur, A. Figotin, Spectra of Random and Almost-Periodic Operators, SpringerVerlag, 1992, 583pp.

[7] L.H. Eliasson, Floquet Solutions for the One-dimensional Quasi-periodic Schrödinger Equation, Comm. Math. Phys. 146 (1992), 3, 447-482.

[8] S. Jitomirskaya, Metal-Insulator Transition for the Almost Mathieu Operator, Ann. of Math. 150 (1999), 1159-1175.

[9] D. Damanik, R. Killip, and D. Lenz, Uniform spectral properties of one-dimensional quasicrystals. III. Alpha-continuity, Commun. Math. Phys. 212 (2000), 191-204. 
[10] B. Simon, Schrödinger Operators in the Twentieth Century, J. Math. Phys. 41 (2000), 6, 3523-3355.

[11] A. Fedotov, F. Klopp, On the Singular Spectrum for Adiabatic Quasi-periodic Schrödinger Operators on the real line, Ann. Henri Poincaré 5 (2004), no. 5, 929978.

[12] A. Fedotov, F. Klopp, On the Absolutely Continuous Spectrum of One-dimensional Quasi-periodic Schrödinger operators in the Adiabatic Limit, Trans. Amer. Math. Soc. 357 (2005), no. 11, 4481-4516.

[13] A. Fedotov, F. Klopp, Strong Resonant Tunneling, Level Repulsion and Spectral Type for One-dimensional Adiabatic Quasi-periodic Schrödinger Operators, Ann. Sci. École Norm. Sup. (4) 38 (2005), no. 6, 889-950.

[14] M.A. Shubin, Density of States for Selfadjoint Elliptic Operators with Almost Periodic Coefficients, Trudy sem. Petrovskii (Moscow University), 3 (1978), 243-281.

[15] J. Bellissard, R. Lima, and E. Scoppola, Localization in n-dimensional incommensurable structures, Commun. Math. Phys. 88, (1983) 465-477.

[16] A.L. Figotin, L.A. Pastur, An Exactly Solvable Model of a Multidimensional Incommensurate Structure, Commun. Math. Physics 95 (1984), 401-425.

[17] J. Bellissard, Almost periodicity in solid state Physics and $C^{*}$-algebras, Mat.-Fys. Medd danske Vid. Selsk. 42 (1989), 3, 35-75.

[18] V. Chulaevsky, E.I. Dinaburg, Methods of KAM theory for Long-Range Quasiperiodic Potentials on $\mathbb{Z}^{\nu}$. Pure Point Spectrum, Commun. Math. Physics 153 (1993), 3, 559-577.

[19] J. Bourgain, M. Goldstein, On Nonperturbative Localization with Quasi-Periodic Potential, Ann. of Math. (2) 152 (2000), 3, 835-879.

[20] J. Bourgain, M. Goldstein, W. Schlag, Anderson Localization on $\mathbb{Z}^{2}$ with QuasiPeriodic Potential, Acta Math. 188 (2002), 41-87.

[21] J. Bourgain, On Quasi-Periodic Lattice Schrödinger Operators, Discrete and Continuous Dynamical Systems 10 (2004), 1\&2, 75-88.

[22] D. Damanik, A Version of Gordon's Theorem for Multidimensional Schrödinger Operators, Trans. Amer. Math. Soc. 356 (2004), 495-507.

[23] L. Parnovski, R. Shterenberg, Complete Asymptotic Expansion of the Integrated Density of States of Multidimensional Almost-periodic Schrödinger Operators, preprint arXiv: 1004.2939v1, to appear in Annals of Mathematics. 
[24] S. Morozov, L. Parnovski, R. Shterenberg, Complete Asymptotic Expansion of the Integrated Density of States of Multidimensional Almost-periodic Pseudo-differential Operators, preprint arXiv: 1204.1076.

[25] V.N. Popov, M.M. Skriganov, Remark on the Structure of the Spectrum of a TwoDimensional Schrödinger Operator with Periodic Potential, Zap. Nauchn. Sem. Leningrad. Otdel. Mat. Inst. Steklov. (LOMI), 109 (1981), pp. 131-133; English transl.: J. Soviet Math., 24 (1984), 2, pp. 239-240.

[26] M.M. Skriganov, A.V. Sobolev, On the Spectrum of a Limit-Periodic Schrödinger Operator, Algebra i Analiz, 17 (2005), no. 5; Engl. Transl.: St. Petersburg Math. J. 17 (2006), 815-833.

[27] Yu. Karpeshina, Y.-R. Lee, Spectral properties of polyharmonic operators with limitperiodic potential in dimension two, D'Analyse Mathématique, 102 (2007), 225-310.

[28] Yu. Karpeshina, Y.-R. Lee, Absolutely Continuous Spectrum of a Polyharmonic Operator with a Limit Periodic Potential in Dimension Two, Communications in Partial Differential Equations, 33 (2008), no. 9, 1711-1128.

[29] Yu. Karpeshina, Y.-R. Lee, Spectral properties of the Schrödinger operator with limit-periodic potential in dimension two, (arXiv:1008.4632v1 [math-ph], pp 1-89), submitted for publication.

[30] G. Gallavotti, Perturbation Theory for Classical Hamiltonian Systems, in Scaling and Self-Similarity in Physics edited by J. Froehlich, Birkhäuser, Basel, Switzerland, 1983, 359-424.

[31] L.E. Thomas, S.R. Wassel, Stability of Hamiltonian systems at high evergy, J. Math. Phys. 33(10), (1992), 3367-3373.

[32] L.E. Thomas and S.R. Wassel, Semiclassical Operators at High Energy, Lecture Notes in Physics, 403 edited by E. Balslev, Springer-Verlag, 1992, 194-223.

[33] J.Frölich, T.Spencer, Absence of Diffusion in the Anderson Tight Binding Model for Large Disorder and Low Energy, Commun. Math. Physics 88 (1983), 2, 151-184.

[34] J. Bourgain, Quasiperiodic Solutions of Hamilton Perturbations of $2 D$ Linear Schrödinger Equation, Ann. of Math. (2), 148 (1998), 2, 363-439.

Department of Mathematics, University of Alabama at Birmingham, 1300 University Boulevard, Birmingham, AL 35294.

e-mail: karpeshi@math.uab.edu; shterenb@math.uab.edu 